\title{
OPEN Detection of SARS-CoV-2 RNA in serum is associated with increased mortality risk in hospitalized COVID-19 patients
}

Diego A. Rodríguez-Serrano ${ }^{1,16 \bowtie}$, Emilia Roy-Vallejo ${ }^{2,16}$, Nelly D. Zurita Cruz ${ }^{3}$ Alexandra Martín Ramírez ${ }^{3}$, Sebastián C. Rodríguez-García ${ }^{4}$, Nuria Arevalillo-Fernández ${ }^{1}$, José María Galván-Román ${ }^{2}$, Leticia Fontán García-Rodrigo ${ }^{3}$, Lorena Vega-Piris ${ }^{5}$, Marta Chicot Llano ${ }^{1}$, David Arribas Méndez ${ }^{6}$, Begoña González de Marcos ${ }^{1}$, Julia Hernando Santos ${ }^{6}$, Ana Sánchez Azofra ${ }^{7}$, Elena Ávalos Pérez-Urria ${ }^{7}$ Pablo Rodriguez-Cortes ${ }^{2}$, Laura Esparcia ${ }^{8}$, Ana Marcos-Jimenez ${ }^{8}$, Santiago Sánchez-Alonso ${ }^{8}$, Irene Llorente ${ }^{4}$, Joan Soriano $0^{7,9}$, Carmen Suárez Fernández ${ }^{2,9}$, Rosario García-Vicuña ${ }^{4}$, Julio Ancochea ${ }^{7,9}$, Jesús Sanz ${ }^{2}$, Cecilia Muñoz-Calleja ${ }^{8,9}$, Rafael de la Cámara ${ }^{10}$, Alfonso Canabal Berlanga ${ }^{1}$, Isidoro González-Álvaro ${ }^{4,16}$, Laura Cardeñoso ${ }^{3,16} \&$ the REINMUN-COVID Group*

COVID-19 has overloaded national health services worldwide. Thus, early identification of patients at risk of poor outcomes is critical. Our objective was to analyse SARS-CoV-2 RNA detection in serum as a severity biomarker in COVID-19. Retrospective observational study including 193 patients admitted for COVID-19. Detection of SARS-CoV-2 RNA in serum (viremia) was performed with samples collected at 48-72 $\mathrm{h}$ of admission by two techniques from Roche and Thermo Fischer Scientific (TFS). Main outcome variables were mortality and need for ICU admission during hospitalization for COVID-19. Viremia was detected in $50-60 \%$ of patients depending on technique. The correlation of $\mathrm{Ct}$ in serum between both techniques was good (intraclass correlation coefficient: $0.612 ; p<0.001$ ). Patients with viremia were older $(\mathrm{p}=0.006)$, had poorer baseline oxygenation $\left(\mathrm{PaO}_{2} / \mathrm{FiO}_{2} i \mathrm{p}<0.001\right)$, more severe lymphopenia $(p<0.001)$ and higher LDH $(p<0.001)$, IL- $6(p=0.021)$, C-reactive protein (CRP; $p=0.022)$ and procalcitonin $(p=0.002)$ serum levels. We defined "relevant viremia" when detection Ct was $<34$ with Roche and $<31$ for TFS. These thresholds had $95 \%$ sensitivity and $35 \%$ specificity. Relevant viremia predicted death during hospitalization (OR 9.2 [3.8-22.6] for Roche, OR 10.3 [3.6-29.3] for TFS; $p<0.001$ ). Cox regression models, adjusted by age, sex and Charlson index, identified increased LDH serum levels and relevant viremia $(H R=9.87$ [4.13-23.57] for TFS viremia and $H R=7.09$ [3.3-14.82] for Roche viremia) as the best markers to predict mortality. Viremia assessment at admission is the most useful biomarker for predicting mortality in COVID-19 patients. Viremia is highly reproducible with two different techniques (TFS and Roche), has a good consistency with other severity biomarkers for COVID-19 and better predictive accuracy.

\footnotetext{
${ }^{1}$ Intensive Care Unit, Hospital Universitario La Princesa, Madrid, Spain. ${ }^{2}$ Internal Medicine Department, Hospital Universitario La Princesa, IIS-IP, Madrid, Spain. ${ }^{3}$ Microbiology Department, Hospital Universitario La Princesa, IIS-IP, Madrid, Spain. ${ }^{2}$ Rheumatology Department, Hospital Universitario La Princesa, IIS-IP, Madrid, Spain. ${ }^{5}$ Methodology Unit, Biomedical Research Institute, Hospital Universitario La Princesa, IIS-IP, Madrid, Spain. ${ }^{6}$ Anaesthesiology Department, Hospital Universitario La Princesa, IIS-IP, Madrid, Spain. ${ }^{7}$ Pneumology Department, Hospital Universitario La Princesa, IIS-IP, Madrid, Spain. ${ }^{8}$ Immunology Department, Hospital Universitario La Princesa, IIS-IP, Madrid, Spain. ${ }^{9}$ Universidad Autónoma de Madrid, Madrid, Spain. ${ }^{10}$ Hematology Department, Hospital Universitario La Princesa, IIS-IP, Madrid, Spain. ${ }^{16}$ These authors contributed equally: Diego A. Rodríguez-Serrano, Emilia Roy-Vallejo, Isidoro González-Álvaro and Laura Cardeñoso. *A list of authors and their affiliations appears at the end of the paper. ${ }^{\circledR}$ email: cancabrilla@hotmail.com
} 
The wide spectrum of COVID-19 severity ranges from asymptomatic to critical cases, albeit less than $10 \%$ of patients develop a severe disease ${ }^{1}$. Even though only a minority of patients need hospitalization for COVID-19, the higher transmission rate of SARS-CoV-2 compared to other viruses, the absence of previous immunity in the population, and the high incidence of this disease in a short period of time are collapsing health care systems worldwide ${ }^{2}$. One remaining challenge from COVID-19 is the difficulty of predicting individual prognosis since determinants of disease severity remain unclear. Previous studies have suggested that age, male sex, obesity, hypertension, and underlying diseases like hematologic malignancies are associated with worse prognosis ${ }^{3-5}$. Likewise, some blood biomarkers are able to predict the emergence of the cytokine storm leading to severe acute respiratory syndrome, the most frequent cause of clinical deterioration in COVID-19 patients ${ }^{6-8}$.

Despite the tropism of SARS-CoV-2 for the upper respiratory tissue ${ }^{9}$, the relevance of its viral load in nasopharyngeal samples remains controversial ${ }^{10}$. However, several authors have reported the detection of SARSCoV-2 RNA in serum or plasma samples (henceforth viremia) associated with a worse prognosis, assessed as higher probability of clinical deterioration, higher levels of interleukin (IL)-6, IL-5 or CXCL10, intensive care unit (ICU) admission, critical disease and death ${ }^{8,11-13}$. Notwithstanding, the detection of viremia with more sensitive techniques was not associated with mortality, but with immune suppression status ${ }^{14}$.

Therefore, we aimed to assess whether there is an association of viremia with COVID-19 severity using two different real-time reverse-transcription polymerase chain reaction (rRT-PCR) techniques, and compare them with other suggested severity biomarkers.

\section{Results}

Demographic and clinical characteristics of the study population. One hundred and ninety-three patients were included; their main demographic and baseline clinical characteristics and laboratory findings are shown in Table 1 and S1 Table.

Depending on the technique, viremia was detected in 95 (48\%; Roche) and 117 (59\%; TFS) patients; the correlation between Ct (Cycle threshold) in serum obtained with both techniques was very good $(r=0.738$ [0.667-0.795], $\mathrm{p}<0.001$ ), and detection by TFS was more sensitive than by Roche (S1A Figure). The agreement to detect viremia was $75.5 \%$ and the intraclass correlation coefficient was $0.612(\mathrm{p}<0.001)$. Conversely, the correlation of Ct between nasopharyngeal and throat swab (NPTS) and serum samples was weak either with TFS or Roche techniques (S1B,C Figure, respectively).

Patients with detectable viremia were older $(\mathrm{p}=0.006)$, with worse baseline arterial oxygen tension/fraction of inspired oxygen ratio $\left(\mathrm{PaO}_{2} / \mathrm{FiO}_{2} ; \mathrm{p}<0.001\right)$, lower lymphocyte count $(\mathrm{p}<0.001)$ and higher $\mathrm{LDH}(\mathrm{p}<0.001)$, IL-6 $(\mathrm{p}=0.021)$, C-reactive protein (CRP; $\mathrm{p}=0.022)$ and procalcitonin $(\mathrm{p}=0.002)$ serum levels compared to patients without detectable viremia. In addition, they were more frequently treated with glucocorticoids $(\mathrm{p}=0.016)$, either oral or bolus ( $\mathrm{p}=0.015)$, and with tocilizumab $(\mathrm{p}=0.001)$ (Table 1 and S1 Table).

There were no significant differences in symptom duration at the time of sample collection between patients with and without viremia ( 9 days [95\%CI 4-13] and 8 [6-11] respectively; $\mathrm{p}=0.223$ ) with both techniques. Neither we observed correlation between disease duration and viral RNA detection in serum with both techniques $(\mathrm{r}=-0.048$ [ $-0.188-0.094], \mathrm{p}=0.510$ for Roche, and $\mathrm{r}=-0.104$ [ $-0.241-0.038], \mathrm{p}=0.151$ for TFS $)$.

Prevalence of SARS-CoV-2 viremia is higher in patients requiring ICU admission. In order to evaluate the relationship between COVID-19 severity and viremia we first studied the relative frequency of viremia in patients who required ICU admission and those who did not. As shown in Fig. 1, viremia was significantly more frequent for both techniques in patients requiring ICU ( $\mathrm{p}<0.001$ for Roche and $\mathrm{p}=0.002$ for TFS). Although TFS was more sensitive to detect viremia, it seemed to be less specific to predict the need for ICU (OR 2.47 [95\% CI 1.37-4.47], $\mathrm{p}=0.003$ for TFS vs 3.04 [1.71-5.44] $\mathrm{p}<0.001$ for Roche).

Additionally, we performed a multivariable logistic regression in order to determine whether viremia can help predicting ICU admission compared to other variables. The best model to predict the need for ICU admission included the following variables: COPD, total lymphocyte count and $\mathrm{PaO}_{2} / \mathrm{FiO}_{2}$ at admission (Table 2). When we forced viremia into this model, it did not reach statistical significance either when it was assessed by Roche or by TFS (Table 2 ).

In addition, when we specifically studied the 86 patients that required ICU admission, no association was found between the presence of viremia and the development of multiorgan dysfunction syndrome prior to admission and during the first $24 \mathrm{~h}$ at the ICU (S2 Table). SARS-CoV-2 viremia may enhance lung damage, leading to the need of ICU admission, while leaving other organs unaffected. Accordingly this parameter is not a good candidate biomarker to predict multiorgan dysfunction.

SARS-CoV-2 viremia predicts mortality in COVID-19 admitted patients. As it was previously suggested that viremia can be associated with a higher risk of mortality, we also analyzed how both techniques predicted death during admission. TFS was more sensitive than Roche's technique but less specific either in the whole population (OR 5.8 [2.6-13.3] for Roche and 3.3 [1.4-7.4] for TFS), or after stratification by requirement of ICU (S2 Figure).

Therefore, we analyzed whether a threshold of viremia (as $\mathrm{Ct}$ ) could help to better predict mortality during hospitalization. As shown in Fig. 2A,B the area under ROC curves for Roche and TFS were quite similar $(0.736$ [0.642-0.829] vs 0.702 [0.603-0.801] respectively; $\mathrm{p}=0.323)$. The best cut-off to predict mortality was $34 \mathrm{Ct}$ for Roche (sensitivity $91 \%$, specificity $38 \%$ ) and 31 Ct for TFS (sensitivity $93 \%$, specificity $32 \%$ ). Using these cut-off points to define "relevant viremia", the capability to predict mortality improved with both techniques: OR 9.2 (3.8-22.6) for Roche and 10.3 (3.6-29.3) for TFS (Fig. 2C,D, respectively). 


\begin{tabular}{|c|c|c|c|c|}
\hline & \multirow[b]{2}{*}{$\begin{array}{l}\text { Study population } \\
(n=193)\end{array}$} & \multicolumn{3}{|l|}{ Viremia } \\
\hline & & $\begin{array}{l}\text { Absent } \\
(n=100)\end{array}$ & $\begin{array}{l}\text { Present } \\
(n=93)\end{array}$ & $\begin{array}{l}\mathbf{P} \\
\text { Value }\end{array}$ \\
\hline Age & $63(55-71)$ & $61(54-68)$ & $67(59-73)$ & 0.001 \\
\hline Male sex & $134(69)$ & $68(68)$ & $66(71)$ & 0.655 \\
\hline Comorbidities & $137(71)$ & $67(67)$ & $70(75)$ & 0.206 \\
\hline Duration of symptoms at admission (days) & $6(4-8)$ & $7(5-9)$ & $6(4-8)$ & 0.120 \\
\hline Baseline $\mathrm{PaO}_{2} / \mathrm{FiO}_{2}$ & $188(100-282)$ & $250(145-348)$ & $146(90-214)$ & $<0.001$ \\
\hline \multicolumn{5}{|l|}{ Treatment during hospitalization } \\
\hline Hydroxychloroquine & $186(96)$ & 97(97) & $89(96)$ & 0.629 \\
\hline Lopinavir/ritonavir & $168(87)$ & $83(83)$ & $85(91)$ & 0.083 \\
\hline Azithromycin & $133(69)$ & $69(69)$ & $64(69)$ & 0.978 \\
\hline Interferon- $\beta$ & $8(4)$ & $2(2)$ & $6(6)$ & 0.121 \\
\hline Glucocorticoids & $134(69)$ & $62(62)$ & $72(77)$ & 0.020 \\
\hline Methylprednisolone bolus & $101(63)$ & $42(53)$ & $59(72)$ & 0.014 \\
\hline Tocilizumab & $91(47)$ & $36(36)$ & $55(59)$ & 0.001 \\
\hline \multicolumn{5}{|l|}{ Laboratory findings } \\
\hline $\begin{array}{l}\text { WBC }\left(10^{3} / \mathrm{mm}^{3}\right) \\
\text { NR: } 4.00-10.00\end{array}$ & $7.18(4.82-9.61)$ & $6.89(4.66-9.35)$ & $7.47(5.00-10.36)$ & 0.374 \\
\hline $\begin{array}{l}\text { Lymphocytes } / \mathrm{mm}^{3} \\
\text { NR: } 1.00-4.00\end{array}$ & $790(590-1165)$ & $950(720-1270)$ & $660(510-860)$ & $<0.001$ \\
\hline $\begin{array}{l}\text { Creatinine }(\mathrm{mg} / \mathrm{dl}) \\
\text { NR:0.70-1.20 }\end{array}$ & $0.86(0.67-1.09)$ & $0.85(0.69-1.03)$ & $0.90(0.63-1.16)$ & 0.563 \\
\hline $\begin{array}{l}\text { LDH (U/L) } \\
\text { NR: 135-225 }\end{array}$ & $390(278-512)$ & $314(246-428)$ & $461(371-565)$ & $<0.001$ \\
\hline $\begin{array}{l}\text { CK (U/L) } \\
\text { NR: } 20-180\end{array}$ & $100(49-270)$ & $94(49-155)$ & $110(56-336)$ & 0.315 \\
\hline $\begin{array}{l}\text { Serum IL-6 (pg/ml) } \\
\text { NR: }<30 \text { pg/ml }\end{array}$ & $20.7(7.9-52.1)$ & $16.4(7.5-41.3)$ & $27.0(7.9-70.3)$ & 0.031 \\
\hline $\begin{array}{l}\text { Ferritin (ng/ml) } \\
\text { NR: } 30-400\end{array}$ & $1542(871-2617)$ & $1418(717-2068)$ & $1637(944-3088)$ & 0.267 \\
\hline $\begin{array}{l}\text { CRP (mg/dL) } \\
\text { NR: } 0.00-0.50\end{array}$ & $12.2(5.5-23.0)$ & $10.3(5.1-19.2)$ & $14.7(7.5-25.7)$ & 0.026 \\
\hline $\begin{array}{l}\text { PCT }(\mathbf{n g} / \mathbf{m l}) \\
\text { NR: } 0.05-0.09\end{array}$ & $0.19(0.11-0.41)$ & $0.13(0.08-0.31)$ & $0.26(0.14-0.56)$ & 0.002 \\
\hline $\begin{array}{l}\text { D-dimer }(\mu \mathrm{g} / \mathrm{ml}) \\
\text { NR:0.14-0.50 }\end{array}$ & $0.78(0.52-1.47)$ & $0.7(0.45-1.22)$ & $0.87(0.67-1.75)$ & 0.030 \\
\hline
\end{tabular}

Table 1. Baseline clinical characteristics of patients according to SARS-CoV-2 RNA detection in blood by Roche technique. Bold values express significant differences between groups. All categorical variables are expressed as number (\%) and quantitative variables as median (p25-75). $\mathrm{PaO}_{2} / \mathrm{FiO}_{2}$ : arterial oxygen tensionfraction of inspired oxygen ratio; WBC: white blood count; NR: normal range; LDH: lactate dehydrogenase; CK: creatin kinase; IL6: interleukin-6; CRP: C-reactive protein; PCT: procalcitonin.
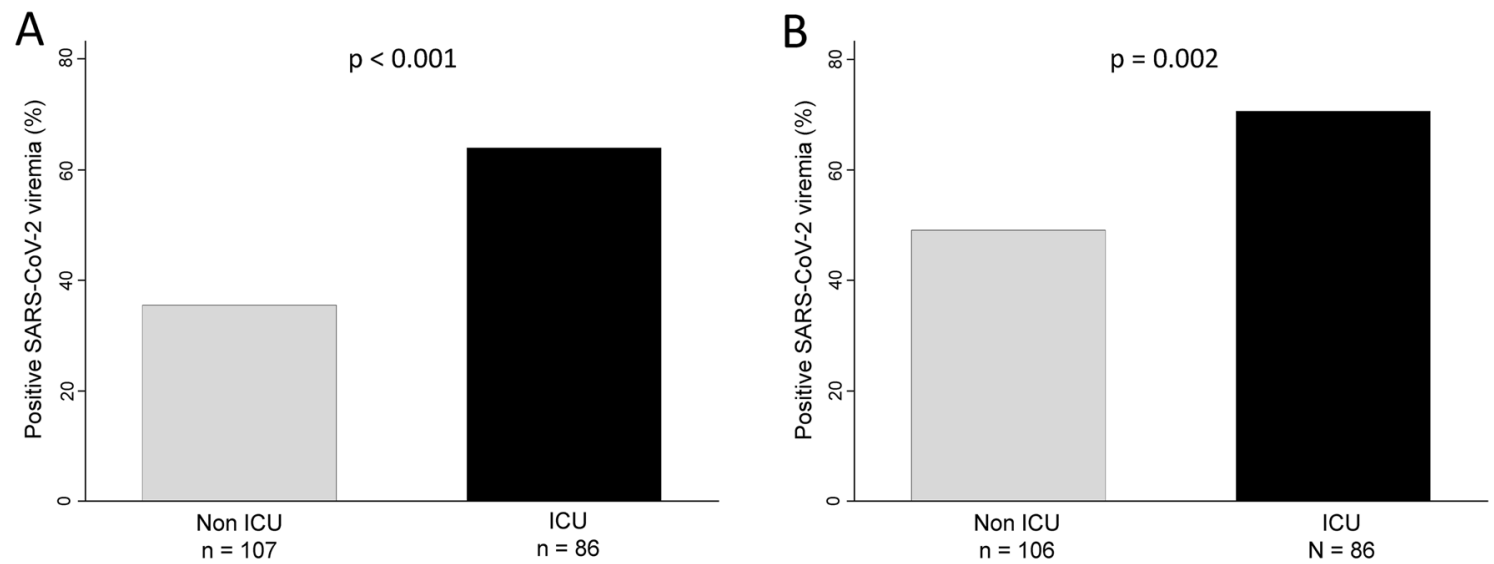

Figure 1. Detection of SARS-CoV-2 RNA in serum (viremia) is more frequent in patients requiring intensive care unit (ICU) admission. (A) Roche technique. (B) Thermo Fisher Scientific technique. Statistical significance was determined with the Chi squared test. For one of the patients TFS determination could not be performed due to lack of sample. 


\begin{tabular}{|l|l|l|l|r|}
\hline \multirow{2}{*}{} & \multicolumn{2}{l|}{ Roche $(\mathbf{n}=\mathbf{1 7 1})$} & \multicolumn{2}{l|}{ Thermo Fisher Systems $(\mathbf{n}=\mathbf{1 7 1})$} \\
\cline { 2 - 5 } & $\mathbf{O R}(\mathbf{9 5} \% \mathbf{C I})$ & $\mathbf{p}$ & OR $(\mathbf{9 5} \% \mathbf{C I})$ & \multicolumn{1}{l|}{ P } \\
\hline COPD & $4.5(0.8-24.2)$ & 0.079 & $4.6(0.8-24.9)$ & 0.076 \\
\hline Low lymphocytes & $1.7(0.9-3.3)$ & 0.130 & $1.5(0.8-3.1)$ & 0.218 \\
\hline Low $\mathrm{PaO}_{2} / \mathrm{FiO}_{2}$ & $4.0(1.8-8.7)$ & $<0.001$ & $4.0(1.9-8.7)$ & $<0.001$ \\
\hline Viremia & $1.7(0.6-4.6)$ & 0.333 & $1.4(0.7-2.9)$ & 0.341 \\
\hline
\end{tabular}

Table 2. Variables predicting the need for Intensive Care Unit admission. COPD: chronic obstructive pulmonary disease; CI: confidence interval; OR: odds ratio; $\mathrm{PaO}_{2} / \mathrm{FiO}_{2}$ : arterial oxygen tension-fraction of inspired oxygen ratio.

A

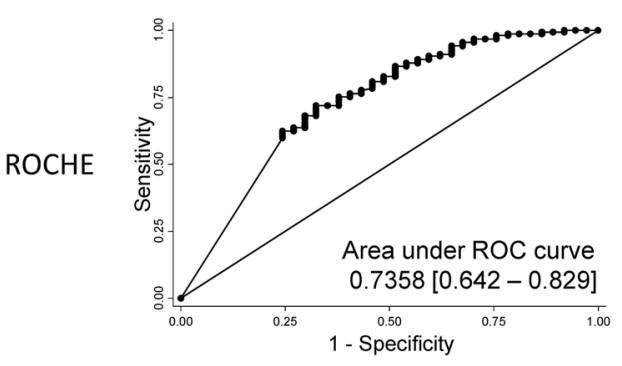

B

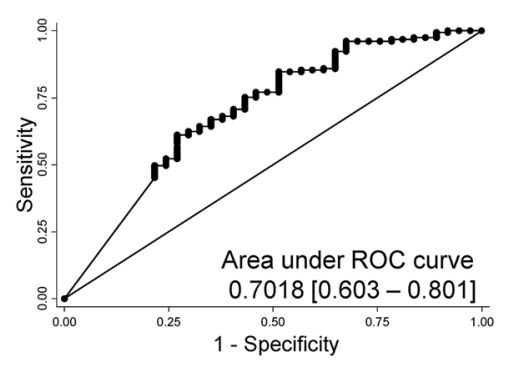

C

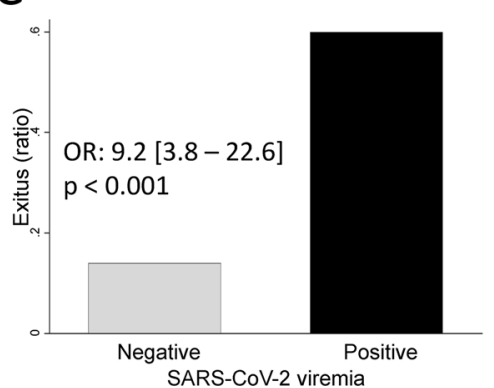

D

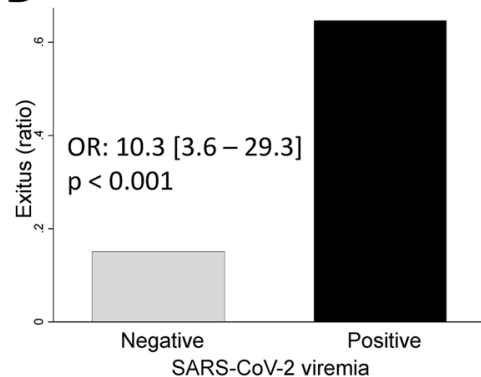

$E$

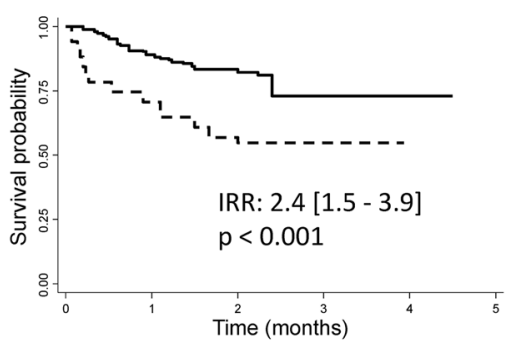

$\mathrm{F}$

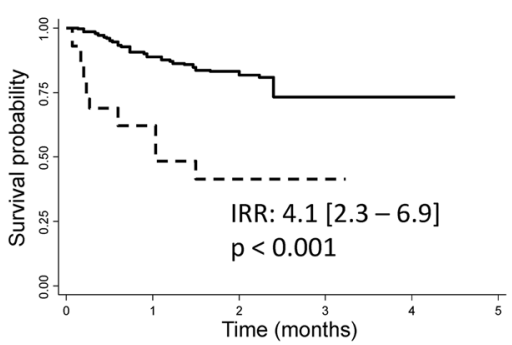

Figure 2. Comprehensive analysis of viremia as a prognostic marker of mortality in patients hospitalized for severe COVID-19. ROC curve-analysis for mortality prediction with Ct values in serum of all patients, according to Roche (A) and Thermo Fisher Scientific [TFS] (B) techniques. Proportion of deceased patients according to the presence (positive) of absence (negative) of relevant viremia determined by Roche (C) and TFS (D) techniques. Survival analysis with Kaplan-Meier estimator of patients hospitalized for COVID-19 who presented (dotted lines) and patients who did not present (solid lines) relevant viremia according to Roche (E) and TFS (F) techniques.

In addition, Kaplan-Meier curves confirmed that those patients with "relevant viremia" at admission survived less than those with non-relevant detection of SARS-CoV-2 RNA (Fig. 2E,F), being slightly higher the incidence risk ratio for viremia determined by TFS compared to Roche (IRR 4.1 [2.3-6.9] vs 2.4 [1.5-3.9] respectively).

SARS-CoV-2 viremia correlates with severity biomarkers previously described in COVID-19 patients. In order to further validate the usefulness of viremia as predictor of mortality in COVID-19, we analyzed its association with clinical and laboratory parameters that have been previously associated with worse outcomes $^{2,7,15}$. As shown in Fig. 3, higher levels of viremia were more frequent in elderly patients $(p=0.011)$ and correlated with higher qSOFA $(p=0.022)$ CURB65 $(p=0.014)$ and Charlson comorbidity $(p=0.078)$ indexes. Furthermore, in addition to SARS-CoV-2 viremia, the prevalence of relevant SARS-CoV-2 viremia also increased with age, with significant differences between patients $<75$ years and $\geq 75$ years (S3 figure).

Regarding laboratory parameters, viremia showed correlation with IL-6 serum levels and lymphocyte count (Fig. 4A,B; $\mathrm{p}=0.014$ and $\mathrm{p}<0.001$, respectively), and a better correlation with lactate dehydrogenase serum levels (Fig. 4C; $\mathrm{p}<0.001$ ). However, lower significant association was observed with ferritin and $\mathrm{D}$-dimer levels (Fig. 4D,E; $\mathrm{p}=0.073$ and $\mathrm{p}=0.023$, respectively).

Finally, we analyzed the hazard ratio for mortality of viremia in comparison with all these parameters. All models were adjusted by age and Charlson index, which significantly affected survival of COVID-19 patients, as well as by sex, which did not significantly affect survival. Baseline qSOFA and CURB65 showed the worst 

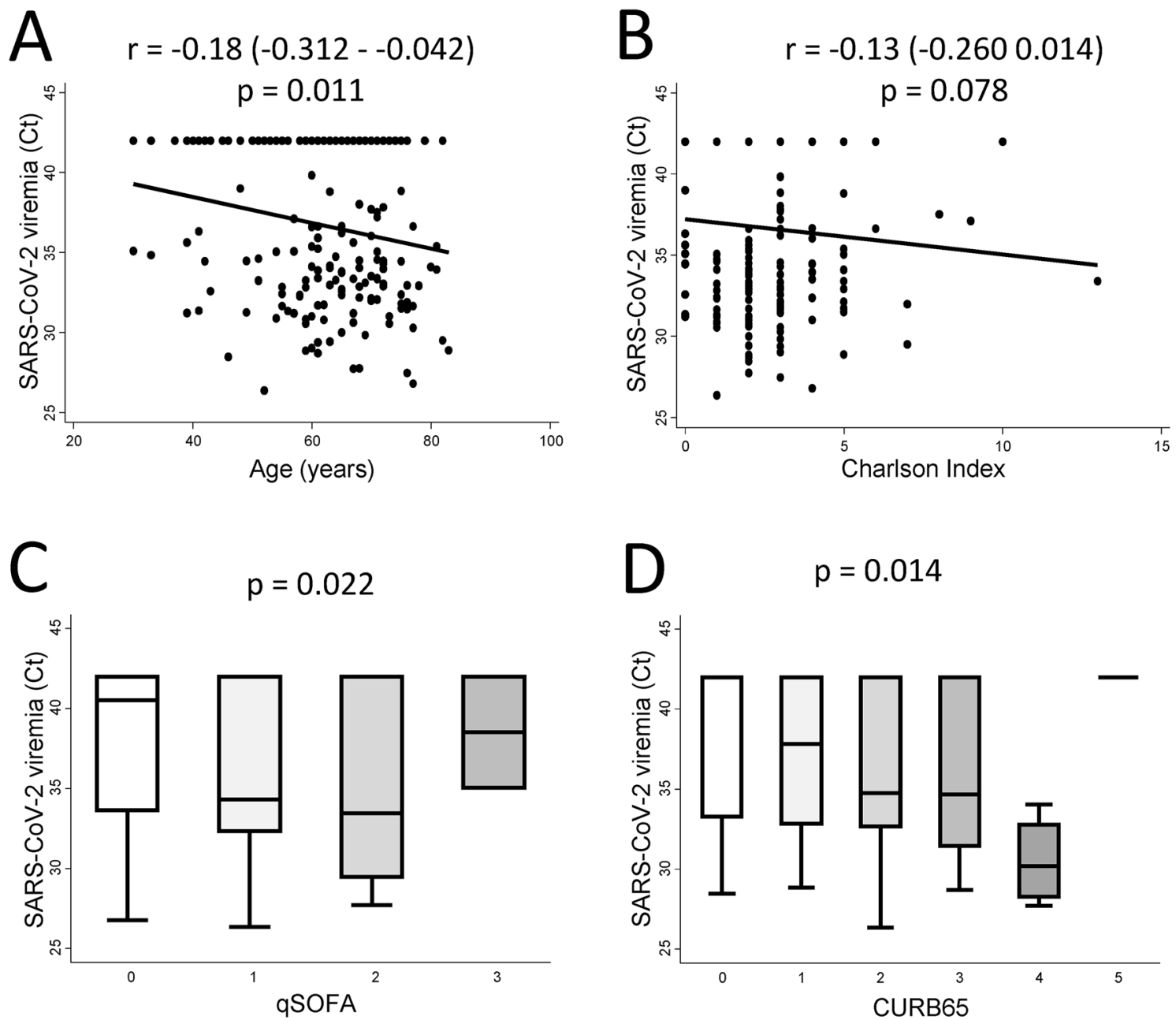

Figure 3. Association between viremia and main sociodemographic and clinical characteristics of patients hospitalized with severe COVID-19. Correlation between viremia levels (Ct value) and (A) age (years) and (B) Charlson comorbidity index (score). Distribution of viremia levels according to qSOFA index (C), and CURB65 scale (D) scores. All Ct data shown here were obtained by rRT-PCR in serum with Thermo Fisher Scientific (TFS) technique. In (A) and (B) data are shown as dot plots of the corresponding values and the fitted linear prediction (black line) obtained with the option lfit of the command twoway of Stata. Correlation coefficients and significance levels were estimated with the Pearson's test. In (C) and (D) data are shown as box-plot representing the interquartile range (p75 upper edge, p25 lower edge, p50 midline in the box), p95 (line above the box), and p5 (line below the box). Significance level was determined with the Kruskal Wallis test.

capability to predict survival (Table 3, model 1). On the contrary, the best predictors always included baseline "relevant viremia" and high LDH serum levels (Table 3, all models). Interestingly, even when relevant viremia, and high LDH levels were included together in model 4, both were able to independently predict survival, although the hazard ratio was higher for the former (Table 3 last two columns; HR 9.87 [4.13-23.57] for TFS viremia and 7.09 [3.39-14.82] for Roche viremia; HRs for high LDH 2.48 [1.21-5.09] with TFS viremia, and 2.39 [1.17-4.90] with Roche viremia).

\section{Discussion}

Many efforts have been made since the first COVID-19 outbreak in China to identify poor prognosis factors in this worldwide and evolving pandemic. Several biomarkers such as low total lymphocyte count, high LDH serum levels, increased acute phase reactants (C-reactive protein, ferritin, fibrinogen, among others) or increased IL-6 serum levels have been proposed ${ }^{6,15}$. Recently, detection of SARS-CoV-2 RNA in blood has been suggested as a potential severity biomarker ${ }^{12,13,16-18}$. In this regard, our results reinforce these previous data with a main contribution to the management of COVID-19: we have determined a semiquantitative threshold for SARS-CoV-2 RNA detection in serum early after admission that allows establishing RNA values ("relevant viremia") associated with higher mortality risk. Furthermore, we have shown that, technically, this finding is reproducible and, it is the most useful biomarker in the clinical setting for predicting mortality in COVID-19 patients.

From a technical point of view, we were able to detect viremia with high accuracy and concordance by using two commercially available kits, from Roche and TFS, as previously described ${ }^{19}$. These kits had been previously approved for detecting SARS-CoV-2 in nasopharyngeal samples. Although the sensitivity of TFS technology was 

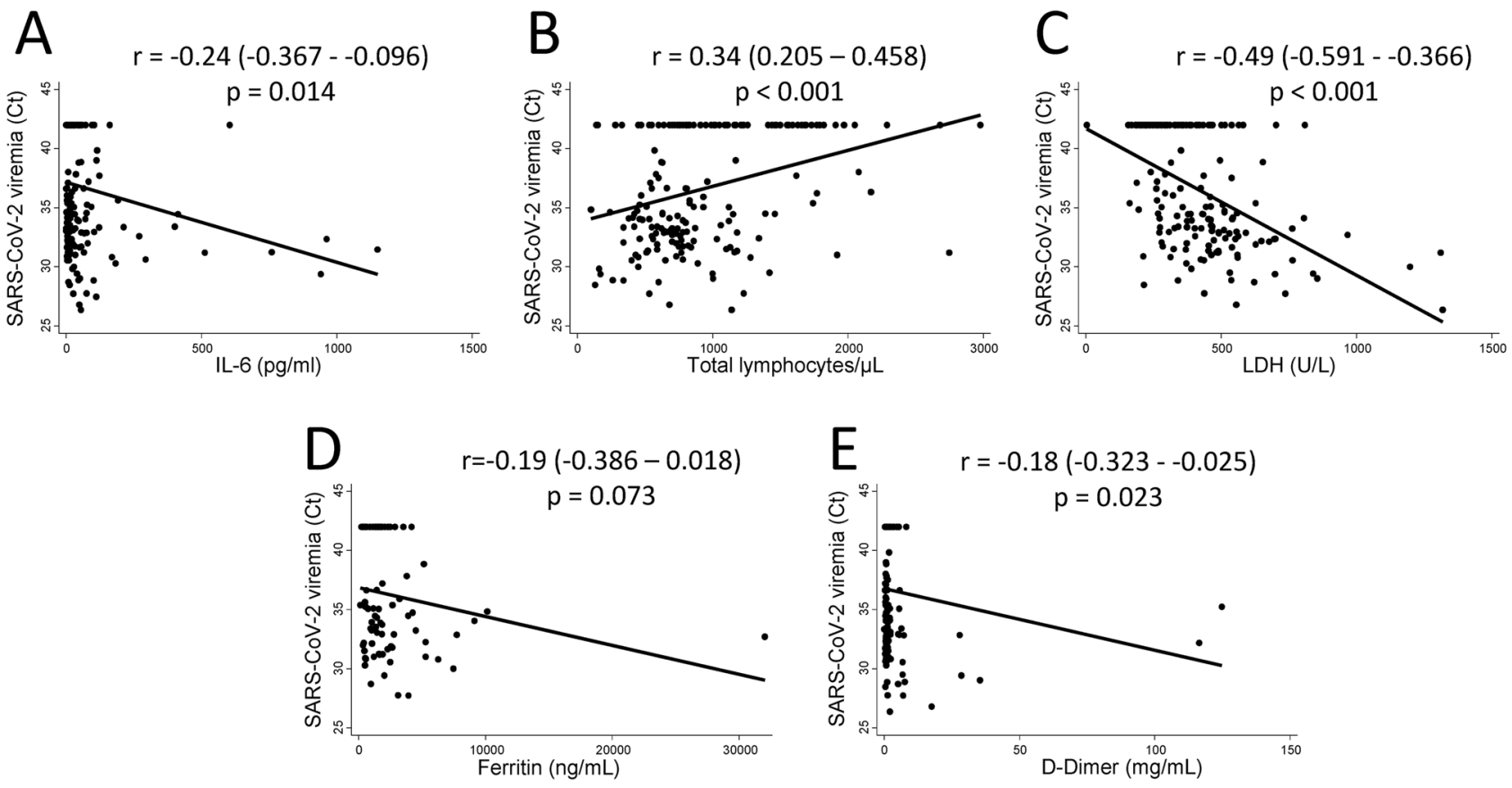

Figure 4. Viremia correlates with the main laboratory prognosis biomarkers described in patients hospitalized with severe COVID-19. Correlation between viremia levels (Ct value by Thermo Fisher Scientific [TFS] technique) and IL-6 levels (pg/mL) (A), lymphocytes count (cel/mm $\left.\mathrm{mm}^{3}\right)(\mathbf{B}), \mathrm{LDH}$ levels $(\mathrm{U} / \mathrm{L})(\mathbf{C})$, ferritin levels $(\mathrm{ng} / \mathrm{mL})(\mathbf{D}), \mathrm{D}-$ Dimer levels $(\mathrm{mg} / \mathrm{mL})(\mathbf{E})$. Data are shown as dot plots of the corresponding values and the fitted linear prediction (black line) obtained with the option lfit of the command twoway of Stata. Correlation coefficients and significance levels were estimated with the Pearson's test.

\begin{tabular}{|l|l|l|l|l|l|l|l|l|}
\hline & \multicolumn{3}{|l}{ Model 1 } & \multicolumn{2}{l}{ Model 2 } & \multicolumn{2}{l|}{ Model 3 } & \multicolumn{2}{l|}{ Model 4 } \\
\cline { 2 - 10 } & HR & $\mathbf{p}$ & HR & $\mathbf{p}$ & HR & $\mathbf{p}$ & HR & P \\
\hline qSOFA & 0.92 & N.S. & N.I. & - & N.I. & - & N.I. & - \\
\hline CURB65 & 1.06 & N.S. & N.I. & - & N.I. & - & N.I. & - \\
\hline Low Ly & 1.85 & 0.080 & - & - & 1.85 & 0.091 & 1.37 & 0.388 \\
\hline High IL-6 & 1.95 & 0.055 & 1.74 & 0.121 & - & - & 1.45 & 0.304 \\
\hline High LDH & 2.74 & 0.005 & 2.74 & 0.008 & 2.57 & 0.011 & - & - \\
\hline Viremia TFS & 13.5 & $<0.001$ & 11.30 & $<0.001$ & 10.4 & $<0.001$ & 9.87 & $<0.001$ \\
\hline Viremia Roche & 8.38 & $<0.001$ & 6.84 & $<0.001$ & 6.69 & $<0.001$ & 7.09 & $<0.001$ \\
\hline
\end{tabular}

Table 3. Mortality predictive value of viremia compared to other factors. CURB65: confusion-urea-respiratory rate-blood pressure-65-years-old index; HR: Hazard Ratio; p: p-value; N.S.: not significant; N.I.: not included; Low Ly.: low total lymphocyte count; IL-6: interleukin-6; LDH: lactate dehydrogenase; qSOFA: quick sepsis related organ failure assessment; TFS: Thermo Fisher Scientific. Model 1 adjusted by age, sex and Charlson index. Model 2 adjusted by age, sex, Charlson index and low total lymphocyte count. Model 3 adjusted by age, sex, Charlson index and high IL-6. Model 4 adjusted by age, sex, Charlson index and high LDH.

slightly better for SARS-CoV-2 RNA detection in serum, this difference disappeared when the specific Ct cut-offs for each kit were applied to define relevant viremia. These results support the notion that the viremia detection can be highly reproducible since the Roche and TFS technologies are based on different procedures for RNA extraction and retrotranscription, as well as different genes for SARS-CoV-2 detection. Furthermore, our results validate previous reports that used the Roche kit and an "in-house" kit for SARS-CoV-2 RNA detection" ${ }^{12,13}$.

From a clinical point of view, we have shown that viremia has a high consistence as a severity biomarker in COVID-19, since it correlates with several variables that have been proposed to be associated with poor evolution in COVID-19, namely old age, comorbidity, qSOFA and CURB-65, as well as with laboratory markers such as high IL-6 or LDH serum levels and severe lymphopenia. Furthermore, multivariable Cox regression analysis identified high LDH serum levels and relevant viremia as independent and solid predictors for mortality after adjusting by age, sex, and presence of comorbidity. Neither qSOFA, CURB-65, low total lymphocyte count, nor high IL-6 serum levels maintained statistically significant association with mortality. Therefore, presence of relevant viremia was proved to be the best mortality predictor in our population since it provides a hazard ratio three times higher than that of the other significant variable, high LDH serum level. 
Certain immunological mechanisms may underlie some of these associations. First, it is tempting to suggest that a decrease in activity or number of immune cells involved in lysis of infected cells may lead to higher viral replication and viral spread in the blood. In this regard, the correlation between IL-6 levels and viremia levels could be related with the recent description of lower cytotoxic potential of NK cells and senescence of CD8+ T lymphocytes associated to high IL-6 levels, which can be restored by tocilizumab treatment ${ }^{20}$. Second, association between lymphopenia and viremia could be related with lymphocyte viral infection and cellular lysis by cytotoxic cells. However, lymphopenia is more likely to be related with increased cellular migration into the lungs, as we have recently described for dendritic cells ${ }^{21}$.

Strengths of our research include: immediacy of results during the emergency situation of the COVID-19 first wave, sufficient sample size $(\mathrm{n}=193)$, fulfillment of STROBE standards for observational research, and a multifaceted view of patients including Immunology, Microbiology and many clinical assessments. However, this study has some limitations. Firstly, a single-center and retrospective design and, therefore, variability on the day of sample extraction. Nevertheless, the average time from beginning of symptoms to sample extraction (median of two days) was equal in patients with positive and negative viremia. Secondly, the moderate specificity of relevant viremia at admission to predict mortality. This finding could be associated to the variability over time of SARS-CoV-2 RNA detection in serum in COVID-19 patients ${ }^{22}$. Because of this variability, viremia detection at a specific time point may not properly reflect the state of viral infection, thereby influencing the assessment of severity outcomes. This could explain why previous descriptions of SARS-CoV-2 RNA detection in blood provided heterogeneous information on its value as severity biomarker ${ }^{13,14,17}$. Therefore, sequential assessment of viremia may be needed to improve its specificity to predict mortality. Last but not least, our findings are restricted to the so-called "first wave", when an overloaded health system could not cover all the needs to provide critical care in some severity ill patients. Although the results are solid, studies in new waves experienced elsewhere are needed to validate their utility throughout the pandemic.

In summary, the study presented here has established the usefulness of SARS-CoV-2 RNA detection in blood in the initial assessment of patients admitted for COVID-19 due to its capability to predict mortality. This assessment would be easily implemented since it is reproducible, regardless of the commercially available kit used for SARS-CoV-2 RNA detection. Accordingly, information from tests widely used for diagnosis could be readily used for prognosis evaluation. However, the need for quantitative standardization of relevant viremia and evaluation of the meaning of persistent Viremia must be addressed in further longitudinal studies.

\section{Methods}

Study design, population and data collection. This is a retrospective observational study of patients admitted to Hospital Universitario La Princesa (HUP) during the early weeks of the first wave of COVID-19 outbreak in Spain (Flow chart in S4 Figure).

Study inclusion criteria were: (a) patients with confirmed detection of SARS-CoV-2 RNA in nasopharyngeal and throat swabs (NPTS), and (b) availability of serum samples at the Microbiology Department for additional measurement of SARS-CoV-2 RNA in blood. Regarding the last criterion, on average, serum samples were obtained at a median of two days from admission (interquartile range [IQR] 1 to 3 ) which nearly corresponded to one week after the beginning of symptoms (median: 8 days, IQR: 6 to 11 ).

Clinical, laboratory and therapeutic data were collected from electronic clinical records and included in an anonymized database as previously described ${ }^{6}$. Baseline clinical data refer to those obtained at admission day and baseline laboratory data refer to one to three days from admission date.

SARS-CoV-2 RNA detection. Nasopharyngeal and throat swab samples were obtained and real-time reverse-transcription polymerase chain reaction (rRT-PCR) was performed with LightCycler Multiplex Virus Master (Roche Diagnostics, USA) using the LightMix Modular SARS and Wuhan CoV E-gene primers (Roche Diagnostics, USA), targeting the $e$ gene as a first-line screening, followed by confirmatory testing with TaqPath COVID-19 CE-IVD Kit RT-PCR (Applied Biosystems, USA) which detects three specific SARS-CoV-2 genomic regions: orf-1ab, $s$, and $n$ genes. Both procedures were performed according to manufacturers' directions on a Quant Studio-5 Real-Time PCR System (Applied Biosystems, USA) and amplification curves were analyzed with QuantStudio Design and Analysis software (Applied Biosystems, USA), for their interpretation by a clinical microbiologist.

To evaluate Viremia, we used $400 \mu \mathrm{L}$ of surplus sera from routine HIV and hepatitis serology assessment that are usually frozen at $-20^{\circ} \mathrm{C}$ for internal quality controls. Serum samples were treated previously for virus inactivation and then tested with two rRT-PCR methods: cobas SARS-COV-2 test (Roche Diagnostics, USA), a qualitative assay for detection of SARS-CoV-2 RNA, and TaqPath COVID-19 CE-IVD RT-PCR Kit (Thermo Fisher Scientific [TFS], USA), a multiplex RT-PCR assay for qualitative detection of SARS-CoV-2 nucleic acids, as previously described ${ }^{19}$. The number of amplification cycles needed for the fluorescence signal to cross the threshold $(\mathrm{Ct})$ was recorded briefly:

(a) Roche test detects a fragment of the orf-1ab region, specific of SARS-COV-2; and a conserved region of $e$ gene, a structural envelope gene, for pan-sarbecovirus detection. Test was performed by cobas 6800 System (Roche Diagnostics, USA); an automatic platform for nucleic acid extraction and RT-PCR amplification and detection. Serum samples were processed according to manufacturer's indications, following the same protocol used for SARS-CoV-2 detection in respiratory samples. Results were analyzed and interpreted automatically by the cobas $6800 / 8800$ Software version 1.02 .12 .1002 .

(b) TFS test detects three specific SARS-CoV-2 genomic regions: orf-1ab, s, and n genes. This technique requires a previous nucleic acid extraction from the sample, which was performed by the automatic eMAG Nucleic 
Acid Extraction System (Biomerieux, France), a validated system for the extraction of nucleic acids from serum and plasma samples. Extraction was carried out according to eMAG manufacturer's directions, obtaining purified nucleic acids in $60 \mu \mathrm{L}$ of elution buffer. Assay was performed using $5 \mu \mathrm{L}$ of the eluted and according to the manufacturer's instructions, by a QuantStudio 5 Real Time PCR System (Applied Biosystems, USA). Amplification curves were analyzed with QuantStudio Design and Analysis software version 2.4.3 (Applied Biosystems, USA). Interpretation of results was carried out by a clinical microbiologist, through amplification curve analysis.

IL-6 serum level measurement. IL-6 serum levels were quantified in duplicate with the Human IL-6 Quantikine high sensitivity enzyme-immune assay from R\&D Systems Europe Ltd. (Abingdon, UK), as described previously.

Variables. We used two different variables to evaluate viremia: (a) dichotomic variable: viremia was considered positive when at least one of the genes studied was positive according to the output of the two systems used; and (b) quantitative variable: when more than one gene target was detected by one test, the mean Ct value of amplified genes was calculated. There was a high consistency among the results of the three genes analyzed with TFS and a slightly lower one for the two genes analyzed in the Roche system ${ }^{19}$. Since none of the two systems (Roche and TFS) provided positive results with $\mathrm{Ct}>40$, by consensus decision, we assigned $\mathrm{Ct}=42$ to those samples reported as negative (for all the genes) by the system.

Baseline IL-6 levels were considered high if $>30 \mathrm{pg} / \mathrm{ml}$, according to their sensitivity and specificity to predict severity determined in a previous study ${ }^{6}$. In addition, in order to be able to compare different biomarkers, other continuous variables previously described as COVID-19 severity biomarkers were also transformed into dichotomic variables considering as cut-off point their median values in our population, namely: total lymphocyte count was considered low when $<700$ cells $/ \mathrm{mm}^{3}$, lactate dehydrogenase (LDH) serum level was considered high when $>400 \mathrm{U} / \mathrm{L}, \mathrm{qSOFA}$ and CURB65 were considered high if $>1$. Patients were considered elderly if age $>70$ years.

Multiorgan dysfunction syndrome was considered when at least two organs reached a score $>1$ in SOFA $\operatorname{scale}^{23}$. Multiorgan failure was considered when at least two organs reached a score $>3$ in SOFA scale ${ }^{24}$.

To analyze whether viremia could predict disease severity, two main outcomes were considered: need for ICU admission and all-cause in-hospital mortality. Only 14 patients out of 89 admitted to ICU did not require invasive mechanical ventilation.

Statistical analysis. Statistical analyses were performed using Stata 14 for Windows (Stata Corp LP, College Station, TX, USA). Quantitative variables following a non-normal distribution were represented as median and IQR, and the Mann Whitney test was used to assess significant differences. Variables with a normal distribution were described by mean \pm standard deviation (SD) and differences between groups were assessed with Student's t-test. Qualitative variables were described as counts and proportions and Chi square or Fisher's exact test was used for comparisons. Correlation between quantitative variables was analyzed using the Pearson correlation test. To estimate the $95 \%$ confidence interval (CI) of correlation coefficients we used the ci2 command of Stata. To evaluate the agreement between viremia assessed by Roche and TFS we estimated the intraclass correlation with the icc command of $\mathrm{R}$.

To determine the variables associated with the need for ICU admission, we performed a multivariable logistic regression analysis that was first modeled by adding all the variables with a p-value lower than 0.15 to the bivariable analysis, namely low lymphocyte count, D-dimer, high $\mathrm{LDH}$, low $\mathrm{PaO}_{2} / \mathrm{FiO}_{2}(<250)$, COPD, high qSOFA, high CURB65, hypertension, C-reactive protein, and high IL-6 level. The final model was reached through backward stepwise removal of variables with p-value higher than 0.15 and using Wald tests to demonstrate that each model was better than its previous iteration. Once the final model was obtained, the dichotomic variables viremia (both Roche and TFS) were forced into the model in order to determine whether they were able to predict the need for ICU admission.

Odds ratio (OR) for mortality according to the presence of viremia was estimated for both systems with the $c s$ command of Stata. Since differences between the two techniques were detected in the sensitivity to detect viremia and the capability of predicting mortality, receiver operating characteristic (ROC) analyses were performed using the roctab command in order to estimate the best cut-off point for each system. Cut-off values for relevant viremia were selected based on the best trade-off values between sensitivity, specificity and the percentage of patients correctly classified.

Survival time was analyzed by Kaplan-Meier method with the sts command of Stata. Date of admission was considered the date of entry, and for exit date we considered the date of death. For those patients without this event, the last revision of the database (electronic chart or telephone call) on May 21st was used to censor their follow-up. Differences in time to death between different variables were analyzed by log-rank test.

To compare the predictive capability of baseline viremia with that of other baseline parameters (qSOFA, CURB65, total lymphocyte count, LDH and IL-6 serum levels) described in previous publications ${ }^{12,13,25}$ we fitted different Cox regression models. In the first model after adjustment by age, sex and Charlson comorbidity index, the hazard ratio (HR) for all other variables including relevant viremia by TFS or Roche was estimated. Since qSOFA and CURB65 did not reach a p-value $<0.15$, they were excluded from the analysis in the subsequent models. Model 2 was adjusted by the same variables included in model 1 and low lymphocyte count, the hazard ratio for the remaining variables was then estimated. Model 3 and 4 were developed as model 2 but substituting low lymphocyte count by high IL-6 and high LDH, respectively. 
Ethics. This study was approved by the Research Ethics Committee of the Hospital Universitario La Princesa, Madrid, (register number 4070) and it was carried out following the ethical principles established in the Declaration of Helsinki. Only oral consent was required as proposed by AEMPS (Agencia Española de Medicamentos y Productos Sanitarios, The Spanish Agency for Medicines and Medical Devices) due to the COVID-19 emergency ${ }^{26}$. All included patients (or their representatives) were informed about the study and gave an oral informed consent (registered in the electronic clinical chart). Patients under 18 years old were not included.

This article was written following the Strengthening the Reporting of Observational Studies in Epidemiology (STROBE) guidelines taking into consideration the difficulties to obtain all needed information in the setting of the COVID-19 pandemic (Appendix).

Received: 20 January 2021; Accepted: 3 June 2021

Published online: 23 June 2021

\section{References}

1. Wiersinga, W. J., Rhodes, A., Cheng, A. C., Peacock, S. J. \& Prescott, H. C. Pathophysiology, transmission, diagnosis, and treatment of coronavirus disease 2019 (COVID-19): A review. JAMA 324, 782-793 (2020).

2. Wu, C. et al. Risk factors associated with acute respiratory distress syndrome and death in patients with coronavirus disease 2019 pneumonia in Wuhan, China. JAMA Intern. Med. 180, 934 (2020).

3. Grasselli, G. et al. Baseline characteristics and outcomes of 1591 patients infected with SARS-CoV-2 admitted to ICUs of the Lombardy region, Italy. JAMA 323, 1574-1581 (2020).

4. Tian, W. et al. Predictors of mortality in hospitalized COVID-19 patients: A systematic review and meta-analysis. J. Med. Virol. 92, 1875-1883 (2020).

5. Vijenthira, A. et al. Outcomes of patients with hematologic malignancies and COVID-19: A systematic review and meta-analysis of 3377 patients. Blood https://doi.org/10.1182/blood.2020008824 (2020).

6. Galván-Román, J. M. et al. IL-6 serum levels predict severity and response to tocilizumab in COVID-19: An observational study. J. Allergy Clin. Immunol. https://doi.org/10.1016/j.jaci.2020.09.018 (2020).

7. Zhou, F. et al. Clinical course and risk factors for mortality of adult inpatients with COVID-19 in Wuhan, China: A retrospective cohort study. Lancet 395, 1054-1062 (2020).

8. Bermejo-Martin, J. F. et al. Viral RNA load in plasma is associated with critical illness and a dysregulated host response in COVID19. Crit. Care 24, 691 (2020).

9. Meyerowitz, E. A., Richterman, A., Gandhi, R. T. \& Sax, P. E. Transmission of SARS-CoV-2: A review of viral, host, and environmental factors. Ann. Intern. Med. https://doi.org/10.7326/M20-5008 (2020).

10. Carmo, A. et al. Clearance and persistence of SARS-CoV-2 RNA in patients with COVID-19. J. Med. Virol. 92, 2227-2231 (2020).

11. Chen, X. et al. Detectable serum severe acute respiratory syndrome coronavirus 2 viral load (RNAemia) is closely correlated with drastically elevated interleukin 6 level in critically ill patients with coronavirus disease 2019. Clin. Infect. Dis. 71, 1937-1942 (2020).

12. Hagman, K. et al. SARS-CoV-2 RNA in serum as predictor of severe outcome in COVID-19: A retrospective cohort study. Clin. Infect. Dis. Off. Publ. Infect. Dis. Soc. Am. https://doi.org/10.1093/cid/ciaa1285 (2020).

13. Veyer, D. et al. Highly sensitive quantification of plasma SARS-CoV-2 RNA shelds light on its potential clinical value. Clin. Infect. Dis. https://doi.org/10.1093/cid/ciaa1196 (2020).

14. Buetti, N. et al. Risk factors for SARS-CoV-2 detection in blood of critically ill patients. Clin. Infect. Dis. https://doi.org/10.1093/ cid/ciaal315 (2021).

15. Kermali, M., Khalsa, R. K., Pillai, K., Ismail, Z. \& Harky, A. The role of biomarkers in diagnosis of COVID-19-A systematic review. Life Sci. 254, 117788 (2020).

16. Hogan, C. A. et al. High frequency of SARS-CoV-2 RNAemia and association with severe disease. Clin. Infect. Dis. https://doi.org/ $10.1093 / \mathrm{cid} / \mathrm{ciaa} 1054(2020)$.

17. Prebensen, C. et al. SARS-CoV-2 RNA in plasma is associated with ICU admission and mortality in patients hospitalized with COVID-19. Clin. Infect. Dis. Off. Publ. Infect. Dis. Soc. Am. https://doi.org/10.1093/cid/ciaa1338 (2020).

18. Xu, D. et al. Relationship between serum SARS-CoV-2 nucleic acid (RNAemia) and organ damage in COVID-19 patients: A cohort study. Clin. Infect. Dis. Off. Publ. Infect. Dis. Soc. Am. https://doi.org/10.1093/cid/ciaa1085 (2020).

19. Ramírez, A. M. et al. Evaluation of two RT-PCR techniques for SARS-CoV-2 RNA detection in serum for microbiological diagnosis. medRxiv https://doi.org/10.1101/2020.11.15.20231795 (2020).

20. Mazzoni, A. et al. Impaired immune cell cytotoxicity in severe COVID-19 is IL-6 dependent. J. Clin. Investig. 130, 4694-4703 (2020).

21. Sánchez-Cerrillo, I. et al. COVID-19 severity associates with pulmonary redistribution of CD1c+DCs and inflammatory transitional and nonclassical monocytes. J. Clin. Investig. 130, 6290-6300 (2020).

22. Kim, J.-M. et al. Detection and isolation of SARS-CoV-2 in serum, urine, and stool specimens of COVID-19 patients from the Republic of Korea. Osong Public Health Res. Perspect. 11, 112-117 (2020).

23. Vincent, J. L. et al. The SOFA (Sepsis-related Organ Failure Assessment) score to describe organ dysfunction/failure. On behalf of the Working Group on sepsis-related problems of the European Society of Intensive Care Medicine. Intensive Care Med. 22, 707-710 (1996).

24. Vincent, J. L. et al. Use of the SOFA score to assess the incidence of organ dysfunction/failure in intensive care units: results of a multicenter, prospective study. Working group on 'sepsis-related problems' of the European Society of Intensive Care Medicine. Crit. Care Med. 26, 1793-1800 (1998).

25. Zheng, S. et al. Viral load dynamics and disease severity in patients infected with SARS-CoV-2 in Zhejiang province, China, January-March 2020: Retrospective cohort study. BMJ 369, m1443. https://doi.org/10.1136/bmj.m1443 (2020).

26. The Spanish Agency for Medicine and Health Products [Agencia Española de Medicamentos y Productos Sanitarios (AEMPS)]. Treatments available for the management of respiratory infection by SARS-CoV-2 [Tratamientos disponibles para el manejo de la infección respiratoria por SARS-CoV-2]. https://www.aemps.gob.es/laAEMPS/docs/medicamentos-disponibles-SARS-CoV-216-4-2020.pdf. Accessed 10 Dec 2020 (2020).

\section{Acknowledgements}

Special thanks to our patients and relatives for agreeing with the use of pseudonymized clinical data and surpluses of clinical samples to perform this study, and PhD Manuel Gomez Gutierrez for his excellent editing assistance. 


\section{Author contributions}

D.A.R-S., E.R-V., S.C.R-G., J.M.G-R., I.G-A. and L.C. conceived this study; N.D.Z.C., A.M.R., L.F.G-R., L.E., A.M-J., S.S-A. and C.M-C. conducted the experiments; N.A-F., M.C-L., D.A.M., B.G.d.M., J.H.S., A.S.A., E.A.PU., P.R-C., I.L., J.B.S., C.S.F., R.G.V., J.A., J.S., R.d.C. and A.C.B. collaborated in the creation of the database and the extraction of data; L.V.P. and I.G-A. analysed the results; D.A.R-S., E.R-V., S.C.R-G., J.M.G-R., J.B.S., R.d.C., I.G-A. and L.C. drafted the work and substantively revised it. All authors reviewed the manuscript.

\section{Funding}

This study was funded with grants: Fondos Supera COVID19 by Banco Santander and CRUE to CS, RG-V, CM and JA; RD16/0011/0012 and PI18/0371 to IGA, from Spanish MINECO and Instituto de Salud Carlos III and co-funded by The European Regional Development Fund (ERDF) A way to make Europe; and co-financed by the Community of Madrid through the Covid 2019 Aid. The work of ER-V has been funded by a Rio-Hortega grant CM19/00149 from the Ministerio de Economia y Competitividad (Instituto de Salud Carlos III) and cofunded by The European Regional Development Fund (ERDF) A way to make Europe. The work of SCR-G has been funded by Fundacion Espanola de Reumatologia. None of these sponsors have had any role in study design; in the collection, analysis, and interpretation of data; in the writing of the report; and in the decision to submit the article for publication.

\section{Competing interests}

The authors of this manuscript have the following competing interests: SCR-G reports grants from Spanish Rheumatology Foundation, during the conduct of the study; nonfinancial support from Roche, Lilly, Pfizer, and Abbvie; personal fees and nonfinancial support from Novartis, Sanofi, and MSD and from UCB-Pharma, outside the submitted work. JA reports grants and personal fees from GlaxoSmithKline and Boehringer Ingelheim; grants from Linde Healthcare; and grants, personal fees, and nonfinancial support from Roche and from Chiesi, outside the submitted work. DAR-S reports personal fees from MSD, outside the submitted work. RdC reports personal fees from MSD, ASTELLAS, Clinigen, Janssen, Roche, and IQONE Health Care outside the submitted work. RG-V reports grants, personal fees, and nonfinancial support from Abbvie, BMS, Lilly, Novartis, Sanofi, Sandoz, and MSD; personal fees from Biogen and Celltrion and from Mylan, outside the submitted work; personal fees and nonfinancial support from Pfizer; grants from Roche; and grants and personal fees from Janssen. CSF reports personal fees from Bayer, BMS, Daichi Sankyo, MSD, and Pfizer, outside the submitted work. CM-C reports competitive grants from ISCIII during the conduct of the study. IG-A reports grants from Instituto de Salud Carlos III, during the course of the study; Personal fees from Lilly and Sanofi; personal fees and nonfinancial support from BMS and Abbvie; research support, personal fees, and nonfinancial support from Roche Laboratories; and nonfinancial support from MSD, Pfizer, and Novartis, not related to the submitted work. The rest of the authors declare that they have no relevant competing interests.

\section{Additional information}

Supplementary Information The online version contains supplementary material available at https://doi.org/ 10.1038/s41598-021-92497-1.

Correspondence and requests for materials should be addressed to D.A.R.-S.

Reprints and permissions information is available at www.nature.com/reprints.

Publisher's note Springer Nature remains neutral with regard to jurisdictional claims in published maps and institutional affiliations.

(i) Open Access This article is licensed under a Creative Commons Attribution 4.0 International License, which permits use, sharing, adaptation, distribution and reproduction in any medium or format, as long as you give appropriate credit to the original author(s) and the source, provide a link to the Creative Commons licence, and indicate if changes were made. The images or other third party material in this article are included in the article's Creative Commons licence, unless indicated otherwise in a credit line to the material. If material is not included in the article's Creative Commons licence and your intended use is not permitted by statutory regulation or exceeds the permitted use, you will need to obtain permission directly from the copyright holder. To view a copy of this licence, visit http://creativecommons.org/licenses/by/4.0/.

\section{the REINMUN-COVID Group}

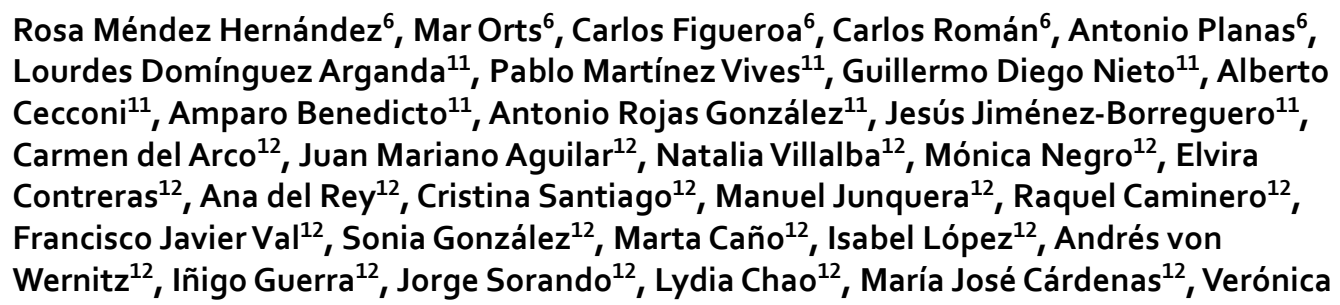


Espiga ${ }^{12}$, Alberto Pizarro ${ }^{12}$, Ángela Figuera Álvarez ${ }^{10}$, Beatriz Aguado ${ }^{10}$, Jimena Cannata ${ }^{10}$, Javier Ortiz ${ }^{10}$, Alberto Morell ${ }^{13}$, Esther Ramírez ${ }^{13}$, Amparo Ibáñez Zurriaga ${ }^{13}$, María Pérez Abanades ${ }^{13}$, Silvia Ruiz García ${ }^{13}$, Tomás Gallego Aranda ${ }^{13}$, María Ruiz ${ }^{13}$, Concepción Martínez Nieto $^{13}$, José María Serra ${ }^{13}$, Francisco Sánchez-Madrid ${ }^{8}$, Ildefonso Sánchez-Cerrillo ${ }^{8}$, Pedro Martínez-Fleta ${ }^{8}$, Celia López-Sanz ${ }^{8}$, Ligia Gabrie ${ }^{8}$, Luciana del Campo Guerola ${ }^{8}$, Elena Fernández ${ }^{8}$, Reyes Tejedor ${ }^{8}$, Pablo A. Patiño Haro ${ }^{1}$, Marina Trigueros Genao ${ }^{1}$, Begoña Quicios Dorado $^{1}$, David Jiménez Jiménez ${ }^{1}$, Macarena Alonso González ${ }^{1}$, Pablo Villamayor ${ }^{1}$, Ignacio de los Santos ${ }^{2}$, Eduardo Sánchez ${ }^{2}$, Fernando Moldenhaver ${ }^{2}$, Pedro Casado², Jose Curbelo², Angela Gutiérrez ${ }^{2}$, Azucena Bautista ${ }^{2}$, Nuria Ruiz Giménez ${ }^{2}$, Angelica Fernández ${ }^{2}$, Lucio García Fraile², Pedro Parra², Berta Moyano², Ana Barrios², Paloma Gil², Iluminada García

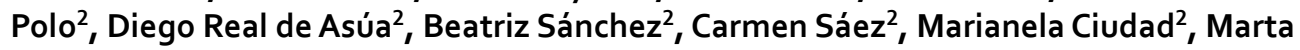
Fernández Rico ${ }^{2}$, Cristina Arévalo Román ${ }^{2}$, Esperanza Morillo Rodríguez ${ }^{2}$, Desiré Navas ${ }^{14}$, María del Carmen Cuevas Torresano ${ }^{3}$, Diego Domingo García ${ }^{3}$, Teresa Alarcón Cavero ${ }^{3}$, Alicia García Blanco ${ }^{3}$, María Auxiliadora Semiglia Chong ${ }^{3}$, Ainhoa Gutiérrez Cobos $^{3}$, Arturo Manuel Fraile Torres ${ }^{3}$, Tamara Alonso ${ }^{7}$, Pedro Landete ${ }^{7}$, Joan Soriano ${ }^{7}$, Carolina Cisneros ${ }^{7}$, Elena García Castillo ${ }^{7}$, Claudia Valenzuela ${ }^{7}$, Francisco Javier García Pérez ${ }^{7}$, Rosa María Girón ${ }^{7}$, Javier Aspa $^{7}$, Celeste Marcos ${ }^{7}$, Enrique Zamora ${ }^{7}$, Gorane Iturricastillo ${ }^{7}$, Mar Barrio Mayo ${ }^{7}$, Encarna Rubia Garrido 7 , Santos Castañeda ${ }^{4}$, Carlos Fernández-Díaz ${ }^{4}$, Eva G. Tomero ${ }^{4}$, Noelia García Castañeda ${ }^{4}$, Ana Ma Ortiz ${ }^{4}$, Cristina Valero ${ }^{4}$, Miren Uriarte ${ }^{4}$, Nuria Montes ${ }^{4}$, Iñigo García Sanz ${ }^{15}$, Francisco Eduardo Viamontes ${ }^{15}$ \& Jesús Delgado Valdueza ${ }^{15}$

${ }^{11}$ Cardiology Department, Hospital Universitario La Princesa, IIS-IP, Madrid, Spain. ${ }^{12}$ Emergency Department, Hospital Universitario La Princesa, IIS-IP, Madrid, Spain. ${ }^{13}$ Hospital Pharmacy, Hospital Universitario La Princesa, IIS-IP, Madrid, Spain. ${ }^{14}$ Medical Biology, Hospital Universitario La Princesa, IIS-IP, Madrid, Spain. ${ }^{15}$ Surgery Department, Hospital Universitario La Princesa, IIS-IP, Madrid, Spain. 